\title{
Report into "on the day cancellations" for plastic surgery in patients who failed to stop their medication
}

\author{
Edward Bass, Parneet Gill \\ Countess of Chester Hospital Trust
}

\begin{abstract}
It was noted that a number of patients were having their procedures cancelled on the day of surgery because their antiplatelet or anticoagulative medications had not been stopped preoperatively. The team recognised that this problem was leading to an unnecessary waste of the department's labour and financial resources, and more importantly was becoming a source of disappointment and anxiety to patients.
\end{abstract}

A retrospective analysis of all plastic surgery cases was performed for procedures listed for the previous 12 months. All cancellations and the reasons for them were recorded, which came to 23 cases. The sum of the financial tariffs for each cancelled procedure was calculated to assess the financial impact of the identified problem: $£ 20,000$. A root cause analysis was performed to assess where this problem was arising in the patient's preoperative journey. The common theme was the lack of information for the gatekeeper regarding the patients' anticoagulant and antiplatelet medications.

A new gatekeeper form was introduced to practice with a subsection specifically highlighting antiplatelet and anticoagulative medications. In addition, this issue was highlighted to the relevant staff in the department.

After four months, a second PDSA cycle was performed in the same manner. Seven cases were cancelled due to anticoagulants or antiplatelets not being stopped prior to surgery. This equated to a net loss of $£ 11,865$ to the department when projected over 12 months, improving on the performance prior to the newly introduced changes.

\section{Problem}

After attending plastic surgery local anaesthetic day case lists at my district general hospital trust in the United Kingdom for several weeks, my supervising registrar and I were concerned by the number of patients who were having their procedures cancelled on the day of surgery because their antiplatelet or anticoagulative medications had not been stopped preoperatively. The team recognised that this problem was leading to an unnecessary waste of the department's labour and financial resources, and more importantly was a source of disappointment and anxiety to patients.

This audit aimed to evaluate the rate of on the day elective plastic surgery cancellations and implemented changes to reduce their impact to our hospital trust.

\section{Background}

On-the-day cancellations of surgical procedures are a long-standing problem for healthcare organisations. They are an unfortunate and avoidable source of waste in both financial and human resources. Indeed, Singhal et al demonstrated that a 10\% cancellation rate from a cohort of 110 patients cost their department $£ 25,881$ in national tariff payments.

Additionally, they cause anxiety and frustration to patients, their carers, and their families. A 2013 study by Dell'Atti concluded that elective surgery cancellations both increase patient anxiety and reduce patients' confidence in their healthcare.

Perhaps surprisingly, the problem is a huge one. A study by Caesar et al of 17,625 consecutive elective orthopaedic procedures found a cancellation rate of $39 \%$; although this is a particularly high rate of cancellation, findings of around $10 \%$ are commonly reported. Interestingly, Caesar et al demonstrated that while the majority of cancellations are due to patient factors, $20 \%$ are directly due to poor preoperative assessment or organisational planning.

Given the above, healthcare professionals should be invested in reducing the elective surgical cancellation rate in their trusts, and strategies to reduce both patient and trust causes of cancellation have been described.

\section{Baseline measurement}

A retrospective analysis of all plastic surgery cases was performed for procedures listed for the previous 12 months. Data were collected from the 'MediTech' software system, which is the management cancellation database and from the coding department. All cancellations and the reasons for them were recorded, and a set standard of a cancellation rate of $8 \%$ was chosen. This was based on a paper by Sanjay et al, published in 2007. 
Forty-two of 6955 total procedures $(0.60 \%)$ were cancelled on the day of surgery. Of these, $23(0.33 \%)$ were cancelled due to anticoagulants or antiplatelets not being stopped. $91 \%$ of patients were booked for theatre via the gatekeeper and $69 \%$ had no information regarding medications on their theatre booking form. The sum of the financial tariffs for each cancelled procedure was calculated at $£ 20,000$.

From these initial results we concluded that our overall elective surgery cancellation rate compared positively with similar reports in the literature. However, given that half of our cancellations were due to medications not being stopped preoperatively - which we considered entirely preventable - and that these still represented a significant financial loss to the department, we decided to pursue a full cycle of quality improvement with a view to improving our cancellation rate and reducing their impact on the department.

See supplementary file: ds3752.jpg - "Pie charts describing the appropriate gathering of patient medications pre-operatively"

\section{Design}

Largely, the problem was a result of the attending surgeon in clinic not inquiring, or not documenting, the patients' medications. As a result, the gatekeeper did not pick this up and patients were not requested to stop the necessary medications.

This issue was highlighted to the relevant staff in the department. In addition, a new gatekeeper form was designed with a subsection specifically highlighting antiplatelet and anticoagulative medications, and guidelines on the need to stop them and for how long prior to treatment. In addition, a section was added regarding high-risk patients that would require specialist advice. The gatekeeper was advised to return the form to the attending surgeon if this subsection was left unfilled. Finally, the initial audit results were presented at a departmental audit meeting to educate junior surgical staff on the issue.

This should be a long-term solution, it has involved minimal changes to everyday practice, and has provided a new booking form which will remain relatively static in design although subject to further quality improvement cycles.

\section{Strategy}

PDSA cycle 1

Our concerns regarding the number of elective surgery cancellations were raised with the audit lead in the department. We planned a retrospective audit of all elective plastic surgery cases in the previous 12 months. We would assess for the overall cancellation rate, the proportion of these that were due to patients not stopping their antiplatelet and anticoagulant medications preoperatively, and the financial impact to the department based on the standard national tariffs. Certain aspects of this would require the expertise of the department's management team and as such they were involved in the project from the outset. Finally, once all the data had been collected, we would design interventions aimed to improve the quality of our healthcare if it was needed.

Once the first audit plan was complete, a retrospective analysis of all plastic surgery cases was performed for elective procedures listed for the previous 12 months using data collected from the 'MediTech' software system, which is the management cancellation database and from the coding department.

All cancellations and the reasons for them were recorded. Forty-two of 6955 total procedures $(0.60 \%)$ were cancelled on the day of surgery. Of these, $23(0.33 \%)$ were cancelled due to anticoagulants not being stopped. Using the standard national tariffs for each cancelled procedure, we calculated the financial impact of these cancellations at $£ 20,000$. A root cause analysis was performed to assess where in the patient's preoperative journey the problem was arising. We noted that although the majority $(91 \%)$ of patients were attending the preoperative gatekeeper, information regarding patient medications was inadequate on the existing theatre booking forms in $69 \%$ of patients and this then led to patients not being informed on which medications to stop and when.

In order to improve the elective plastic surgery cancellation rate, several interventions were enacted. Firstly, the issue was highlighted to the relevant staff in the department at a department audit meeting to educate the relevant staff on the issue and where we were falling short. In addition, a new theatre booking form was designed with a subsection specifically highlighting antiplatelet and anticoagulative medications and guidelines on the need to stop them and for how long prior to treatment. A particular section was added regarding high-risk patients who would require specialist advice. The gatekeeper was advised to return the form to the attending surgeon if this subsection was left unfilled. Finally, the departmental staff were informed of these changes and copies of the new booking form were disseminated amongst departmental staff. A provisional date was set to repeat the quality improvement cycle four months following the introductions of these interventions.

\section{PDSA cycle 2}

The second improvement cycle aimed to assess the impact of the improvement interventions enacted from PDSA cycle 1. In much the same manner, we would assess the previous four months of elective plastic surgical cases for the overall cancellation rate, which were due to patients not stopping their anticoagulants and antiplatelet medications and the financial impact of these cancellations on the department.

As such, a retrospective analysis of all plastic surgery cases was performed for procedures listed for the previous four months. As previously, data was collected from the 'MediTech' software system, the management cancellation database and from the coding department. All cancellations and the reasons for them were recorded. The aim was to improve from on the department's performance prior to the intervention.

We found nine cases $(0.47 \%)$ were cancelled, of which seven $(0.37 \%)$ were due to anticoagulants or antiplatelets not being 
stopped preoperatively. All patients were booked via the gatekeeper and $55 \%$ of cancellations had inadequate medication information on the theatre booking forms. Using the standard national tariffs for each cancelled procedure, the financial impact of the cancellations over the previous four months was calculated at £3955. Over 12 months this equates to a net loss to the department of $£ 11,865$. A second root cause analysis of each cancelled case revealed that these patients were being seen in one of the department's satellite clinics and the booking forms being filled out either remotely or in retrospect. Because of this, patient medication data was inadequate so they were not being advised to stop certain medication preoperatively.

Given these findings, the updated booking form was introduced to the department's satellite clinic sites. This meant that they could be filled out at the time of seeing the patient in clinic and then sent directly to the gatekeeper with adequate medication information for the correct preoperative information to be given to the patient. A provisional date was set to perform another audit cycle in a further four months.

\section{Results}

Following the introduction of our improvement interventions nine elective cases $(0.47 \%)$ were cancelled over the four month period, of which seven $(0.37 \%)$ were due to anticoagulants or antiplatelets not being stopped prior to surgery. All nine were booked via the theatre gatekeeper and of these five $(55 \%)$ had inadequate information regarding medications on the theatre booking form. The sum of the financial tariffs for each cancelled procedure was calculated to assess the financial impact of the identified problem: $£ 3955$. Projecting forward, this figure suggests that over 12 months there would be a total net loss to the department of $£ 11,865$.

Perhaps strangely, this demonstrates that although the elective surgery cancellation rate due to patients not stopping antiplatelet and anticoagulant medications did not improve significantly, the financial impact of the cancellations improved by $41 \%$, as did the documentation of antiplatelet or anticoagulant medications being taken by the patients. We have hypothesised that the new theatre booking form not being introduced to the department's satellite clinics after PDSA cycle 1 explains these results. This has been addressed in PDSA cycle 2 and we would estimate a significant improvement in the cancellation rate following PDSA cycle 3.

See supplementary file: ds3753.pdf - "The new theatre booking form encompassing changes"

\section{Lessons and limitations}

A number of lessons were learned from carrying out this process. Firstly, great efforts were made prior to the first audit cycle to gain support for a change from both surgical and managerial stakeholders. This made the institution of any changes to the theatre booking protocol far easier. Also, we learnt that in a department with multiple peripheral clinic sites it is important to introduce the changes to each site to maximise the service improvements. This process has demonstrated that repetitive evaluation of department performance in activities that are generally seen mundane can be easily undertaken and make real differences as part of clinical governance and quality improvement.

There were limitations to the process. Firstly, the re-audit cycle was shorter than the first cycle and secondly, the process could have been affected by the six-monthly changes in surgical trainees. In addition, there was a delay in the introduction of the updated booking form to the peripheral clinic sites, however, this has since been resolved and a further quality improvement cycle will be performed in four months time.

\section{Conclusion}

Introducing the changes to the theatre booking process appears to have been partially successful. The number of elective surgical cancellations has not reduced significantly however; the financial impact of cancellations on the trust has reduced by $41 \%$.

The failure to reduce the cancellation rate is likely due to overlooking the impact of the department's satellite clinics on the total number of elective surgery cases, and the failure to introduce all the necessary interventions to these clinics. Following subsequent application of these improvement interventions at these clinics, we expect to see improvements in both financial impact and cancellation rate after further quality improvement cycles.

\section{References}

Singhal R, Warburton T, Charalambous CP. Reducing same day cancellations due to patient related factors in elective orthopaedic surgery: experience of a centre in the UK. J Perioper Pract. 2014 Apr;24(4):70-4.

Dell'Atti L. The cancelling of elective surgical operations causes emotional trauma and a lack of confidence: study from a urological department. Urologia. 2013;0(0):0.

Caesar U, Karlsson J, Olsson LE, Samuelsson K, HanssonOlofsson $\mathrm{E}$. Incidence and root causes of cancellations for elective orthopaedic procedures: a single center experience of 17,625 consecutive cases. Patient Saf Surg. 2014;8:24.

Sanjay P, Dodds A, Miller E, Arumugam PJ, Woodward A. Cancelled elective operations: an observational study from a district general hospital. Journal of Health Organisation and Management. 2007;(21):54-58

Dimitriadis PA, lyer S, Evgeniou E. The Challenge of cancellations on the day of surgery. The International Journal of Surgery. 2013;11(10):1126-30.

\section{Declaration of interests}

There are no conflicts of interest to declare 


\section{Acknowledgements}

This project is intended for the Agents for Change Conference 2014.

Charlotte Walton, Mr Ali Juma 